\title{
Multilayer based interferential-plasmonic structure: metal cluster 3D grating combined with dielectric mirror
}

\author{
V. Janicki ${ }^{1 *}$, J. Sancho-Parramon ${ }^{1}$ and H. Zorc ${ }^{1}$ \\ ${ }^{1}$ Ruđer Bošković Institute, Bijenička c. 54, 10000 Zagreb, Croatia \\ *corresponding author: janicki@irb.hr
}

\begin{abstract}
A three dimensional (3D) photonic microstructure consisting of metal clusters embedded in dielectric matrix is coated with a dielectric mirror. The produced photonic structure shows optical behaviour that combines the interferential effects of the multilayer stack and the surface plasmon resonance of metal clusters. Due to its feasibility and the possibility to widely modify the optical properties of the resulting interferential-plasmonic structure, this approach represents a promising method for the production of novel optical components.
\end{abstract}

\section{INTRODUCTION}

Metal clusters attract lot of interest due to the effect of surface plasmon resonance of free electrons, showing a strong absorption at specific wavelengths that depend on the particle size, shape and spatial distribution ${ }^{1}$. They are used in selective absorbers, optical polarizers and data storage ${ }^{2-4}$, or in chemical and biological sensing and surface enhanced spectroscopy ${ }^{5,6}$. Metal clusters in dielectric matrix or present on the surface of a substrate can be structured by electric field assisted dissolution (EFAD) ${ }^{7}$. Simultaneous application of electric field and increased temperature induces dissolution of metal clusters. Using patterned electrodes EFAD can be applied locally, giving mirrored pattern on the sample containing metal clusters. Due to the possibility of structuring, the application can be extended to the production of devices ranging from plasmonic waveguides ${ }^{8}$ to gratings ${ }^{9}$.

We have fabricated 3D microstructure by EFAD of metal clusters embedded in multilayer stacks ${ }^{10,11}$. The optical behaviour of the structure is additionally tailored by the interferential effects: the stack is further coated with a dielectric mirror. The obtained structure shows the tailored optical behaviour of the resulting 3D structure and the interference phenomenon that takes place within the multilayer stack.

\section{EXPERIMENTAL}

A multilayer structure of metal clusters embedded in dielectric matrix was prepared by electron beam evaporation of $\mathrm{Ag}$ and $\mathrm{SiO}_{2}$ at $\mathrm{N}-\mathrm{BK} 7$ glass substrates of $1 \mathrm{~mm}$ thickness. The structure of the coating was glass $/\left(\mathrm{Ag} / \mathrm{SiO}_{2}\right)^{10}$, with layer mass thickness $7 \mathrm{~nm}(\mathrm{Ag})$ and $20 \mathrm{~nm}\left(\mathrm{SiO}_{2}\right)$. Deposition rates were $2-3 \AA / \mathrm{s}$ for $\mathrm{Ag}$ and $10 \AA / \mathrm{s}$ for $\mathrm{SiO}_{2}$. There was no pre-heating of the substrates. Ag film deposited in this conditions remains in the shape of metal clusters with no significant percolation among the clusters. The $\mathrm{SiO}_{2}$ layers of the given thickness cover the metal nanoclusters completely, so the resulting coating has a real multilayer structure ${ }^{11,12}$.

The prepared samples were mounted between electrodes and exposed to EFAD for 2 hours at $250{ }^{\circ} \mathrm{C}$ and $1000 \mathrm{~V}$. One part of the anode was a diffracting grating, the other part was made as uniform contact and a part of the sample has not been in contact with the anode ${ }^{10}$.

Upon the treatment, the sample has been returned to the deposition chamber and a dielectric $\mathrm{TiO}_{2} / \mathrm{SiO}_{2}$ quarter wave $\mathrm{H}(\mathrm{LH})^{3}$ mirror centered at $450 \mathrm{~nm}$ has been deposited.

The measurements of optical performances of different parts of the samples were done by Perkin Elmer 
Lambda 25 spectrophotometer and Woollam VWASE ellipsometer. Reflectance and transmittance measurements were performed in the range 350-1100 nm, each $1 \mathrm{~nm}$, and ellipsometric measurements of $\psi$ and $\Delta$ in the range $0.57-4.35 \mathrm{eV}$, each $0.02 \mathrm{eV}$ at $45^{\circ}, 60^{\circ}$ and $75^{\circ}$ incidence angle. In addition to this, diffraction patterns of generated structures were measured with ellipsometer working in scatterometry mode. The incidence beam was coming to the uncoated side of the sample. Transmittance and reflectance in the range $300-1100 \mathrm{~nm}$, each $25 \mathrm{~nm}$, at the scattering angles $0-10^{\circ}$ and $20-30^{\circ}$, respectively, by $0.2^{\circ}$.

\section{RESULTS AND DISCUSSION}

Optical performance of as deposited (AD) samples is dominated by surface plasmon resonance of metal clusters, showing maximum of absorption in the range 350-480 nm (Figure 1.). It is possible to distinguish three different parts of the sample after the treatment. Zone A is the transparent part where metal clusters are dissolved due to EFAD. The spectrum shows interferential fringes that can be related to the refractive index difference of $\mathrm{SiO}_{2}$ layers and voids remaining after dissolution of layers containing metal clusters (Figure 1.). The significant absorption (A) in the visible and UV part can also be related to the voids, i.e. representing losses due to scattering of the light at voids. Zone B was not in the contact with the anode during the treatment, so it was exposed to the increased temperature only. The optical spectra show no significant difference corresponding to the AD sample (Figure 1.). The absorption peak is somewhat narrower and more intense after annealing that can be related to the thermally induced changes in shape and/or size of nanoclusters ${ }^{10}$.

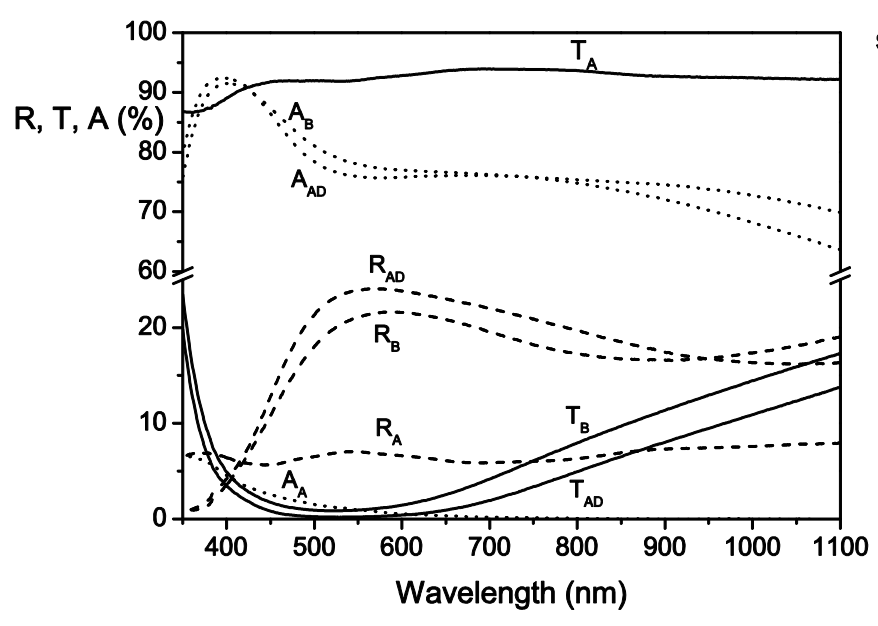

Figure 1. Reflectance (dashes), transmittance (full line) and absorption (dots) of the $\mathrm{AD}$ sample and zones of the sample where metal nanoclusters were dissolved (A) and where they were annealed only (B). The measurements were taken at $0^{\circ}$ angle of incidence.

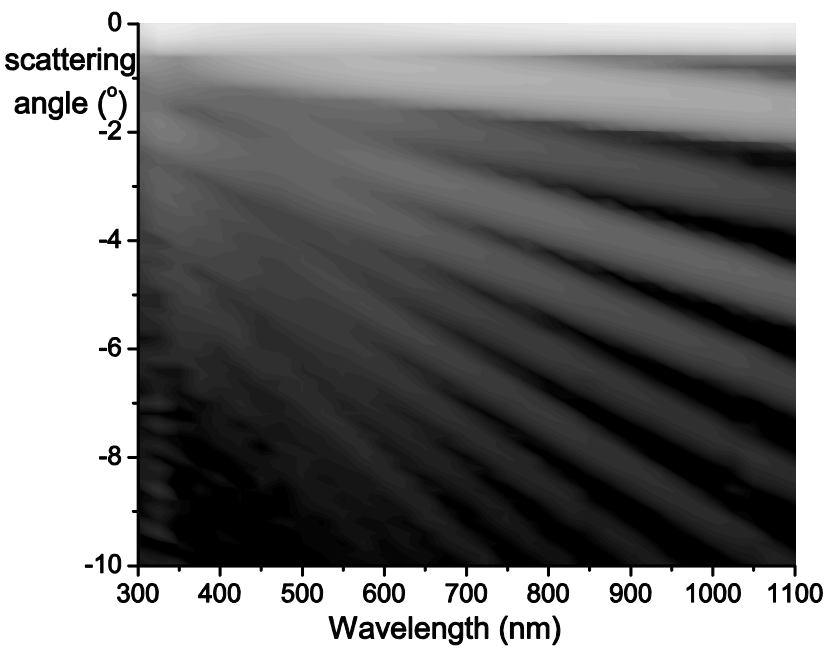

Figure 2. Diffraction transmittance pattern in logarithmic scale of zone C (diffraction grating) of the treated sample, in respect to the wavelength and scattering angle. It is possible to see different diffraction orders and their intensities. The incidence beam comes from the uncoated side of the sample.

Zone $\mathrm{C}$ is the one with the EFAD imprinted structure of diffraction grating from the anode. This planar structure consisting of stripes with dissolved and non-dissolved metal clusters is repeated throughout the coating forming in this way a 3D structure. The diffraction pattern is presented in Figure 2. showing the efficiency of the grating in transmittance in respect to the wavelength and scattering angle. The efficiency is higher for the wavelengths 
where $T_{B}$ is lower $(500-600 \mathrm{~nm})$.

The dielectric mirror has been deposited onto the treated sample and a bare glass substrate. Optical measurements of zones A, B and C were performed. Optical performance of the final structure is presented in Figure 3. and Figure 4.

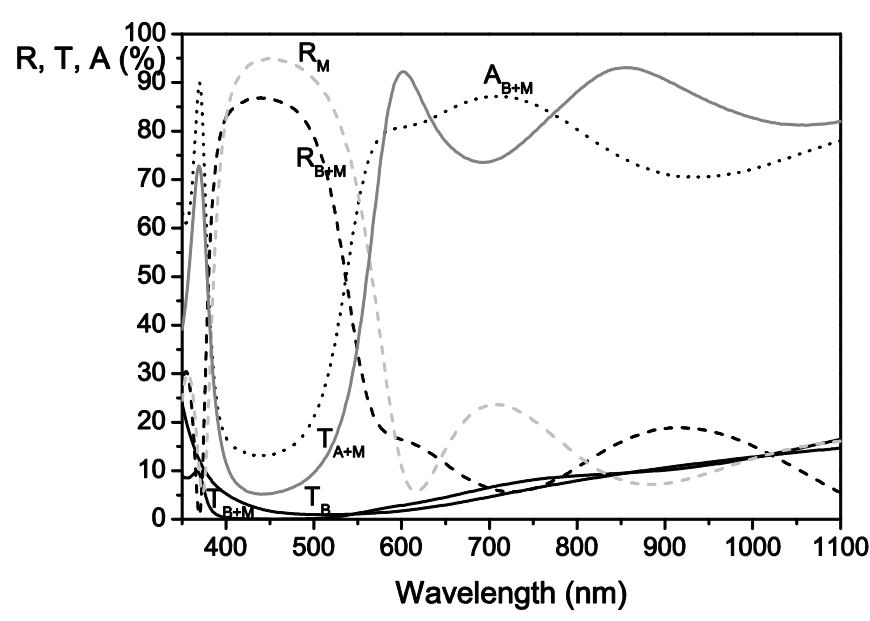

Figure 3. Reflectance (dashes), transmittance (full line) and absorption (dots) of the mirror $\left(\mathrm{R}_{\mathrm{M}}\right)$, sample before deposition of mirror $\left(\mathrm{T}_{\mathrm{B}}\right)$ and zones $\mathrm{A}$ and $\mathrm{B}$ after deposition of mirror. The measurements were taken at $0^{\circ}$ angle of incidence.

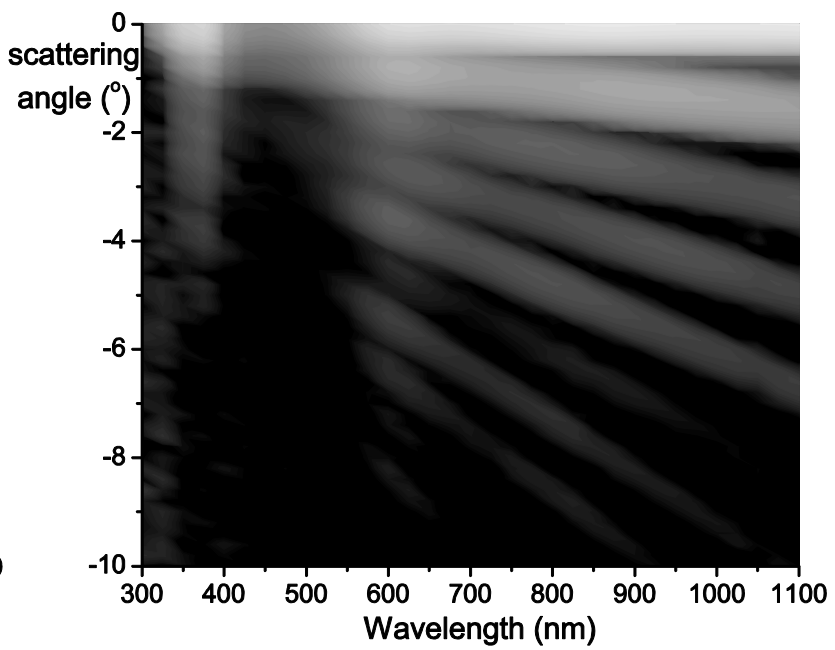

Figure 4. Diffraction transmittance pattern in logarithmic scale of zone C (diffraction grating) of the sample with mirror, in respect to the wavelength and scattering angle. It is possible to see the reduced efficiency of the grating in the range 390-540 $\mathrm{nm}$. The incidence beam comes from the uncoated side of the sample.

Reflectance and transmittance of zone A, same as reflectance of zone B, are dominated by the performance of the mirror itself, that reflectance $\left(\mathrm{R}_{\mathrm{M}}\right)$ is shown for comparison as well. In the case of incidence light coming to the coated side, high proportion of the intensity is reflected, so less intensity is absorbed in the comparison to the sample without the mirror. Therefore, the absorption of the zone $B\left(A_{B}\right)$ is following opposite trend than reflectance $\mathrm{R}_{\mathrm{B}+\mathrm{M}}$. In the range below $400 \mathrm{~nm}$ absorption of $\mathrm{TiO}_{2}$ contributes as well. The resulting transmittance $\left(\mathrm{T}_{\mathrm{B}+\mathrm{M}}\right)$ is finally lower than the one for the sample without the mirror. It is also possible to see that $\mathrm{T}_{\mathrm{A}}$ and $\mathrm{T}_{\mathrm{B}}$, that correspond to the transmittance of the lines and space between them in the diffraction grating, both have low values between 390 and $540 \mathrm{~nm}$. This reduces the performance of the grating that is clearly seen in Figure 4 . Additional diffraction orders maxima are present due to the interference effects.

\section{CONCLUSIONS}

3D microstructure has been obtained by EFAD of metal clusters embedded in multilayer stacks. The parts of the stack containing metal clusters i.e. where electric field dissolution was not performed show absorption due to surface plasmon resonance, while the rest of the stack is transparent. The stack is further coated with a dielectric mirror. Thus, in addition to the SPR-based metallodielectric properties, the optical behaviour of the structure is refined by the interferential effects. The obtained structure shows the tailored optical behaviour of the resulting 3D structure and the interference phenomenon that takes place within the multilayer stack. 
From a technological point of view these 3D structures can be fabricated easily, as their production requires the combination of well established thin film deposition processes and the EFAD technique. Multilayer thin film stacks enable the design of a wide variety of components with different optical performances: antireflective coatings, band-pass filters, mirrors, polarisers etc. The production of these interferential-plasmonic structures could be done at low cost and easily implemented in mass-production processes, as it requires well-established thin film deposition technology and inexpensive EFAD technique.

\section{ACKNOWLEDGEMENT}

The authors would like to thank to Mr. Zdravko Dundović for preparing the original diffraction grating.

\section{REFERENCES}

1. Kreibig, U. and M. Vollmer, Optical Properties of Metal Clusters, Springer Series in Material Science, Springer, Berlin, 1995.

2. Kachan, S., O. Stenzel and A. Ponyavina, "High-absorbing gradient multilayer coatings with silver nanoparticles", Appl. Phys. B: Lasers Opt., Vol. 84, 281-287, 2006.

3. Gotschy, W., K. Vonmetz, A. Leitner and F. R. Aussenegg, "Optical dichroism of lithographically designed silver nanoparticle films", Opt. Lett., Vol. 21, 1099-1101, 1996.

4. Yih, J.-N., W.-C. Hsu, S.-Y. Tsai and S.-J. Chen, "Enhanced readout signal of superresolution near-field structure disks by control of the size and distribution of metal nanoclusters", Appl. Opt., Vol. 44, 3001-3005, 2005.

5. Lahav, M., A. Vaskevich and I. Rubinstein, "Biological Sensing Using Transmission Surface Plasmon Resonance Spectroscopy”, Langmuir, Vol. 20, 7365-7367, 2004.

6. Sockalingum, G. D., A. Beljebbar, H. Morjani, J. F. Angiboust and M. Manfait, "Characterization of island films as surface-enhanced Raman spectroscopy substrates for detecting low antitumor drug concentrations at single cell level", Biospectroscopy, Vol. 4, S71-S78, 1998.

7. Podlipensky, A., A. Abdolvand, G. Seifer, H. Graener, O. Deparios and P.G. Kazansky, "Dissolution of Silver Nanoparticles in Glass through an Intense DC Electric Field”, J. Phys. Chem. B, Vol. 108, 17699-17702, 2004.

8. Krenn, J. R., 'Nanoparticle waveguides: Watching energy transfer”, Nat. Mater., Vol. 2, 210-211, 2003.

9. Wedge, S. and W. L. Barnes, "Surface plasmon-polariton mediated light emission through thin metal films", Opt. Express, Vol. 12, 3673-3685, 2006.

10. Sancho-Parramon J., V. Janicki, J. Arbiol, H. Zorc and F. Peiro, "Electric field assisted dissolution of metal clusters in metal island films for photonic heterostructures", Appl. Phys. Lett., Vol. 92, 163108-1-163108-3, 2008.

11. Janicki V., J. Sancho-Parramon, F.Peiró and J. Arbiol, "Three-dimensional photonic microstructures produced by electric field assisted dissolution of metal nanoclusters in multilayer stacks", Appl.Phys. B, Vol. 98, 93-98, 2010.

12. Lončarić, M., J. Sancho-Parramon, M. Pavlović, H. Zorc, P. Dubček, A. Turković, S. Bernstorff, G. Jakopic, and A. Haase, "Optical and structural characterization of silver islands films on glass substrates", Vacuum, Vol. 84, 188-192, 2010. 